\title{
Estudio neuropsicológico de la dismorfia muscular y del trastorno obsesivo- compulsivo*
}

Neuropsychological Study of Muscle Dysmorphia and ObsessiveCompulsive Disorder

\section{María Alejandra Favila Figueroa ${ }^{\mathrm{a}}$}

Universidad Autónoma del Estado de México, México ORCID: http://orcid.org/0000-0001-9386-952X

Teresa Contreras Troya

Universidad Autónoma del Estado de México, México

SAlvador JuÁrez López

Universidad Autónoma del Estado de México, México

Gabriela Aldana González

Universidad Autónoma del Estado de México, México Alejandra Valencia Cruz

Universidad Nacional Autónoma de México, México

a Autor de correspondencia. Correo electrónico: mafavilaf@uemex.mx

Para citar este artículo: Favila Figueroa, M. A., Contreras Troya, T., Juárez López, S., Aldana González, G., \& Valencia Cruz, A. (2018). Estudio neuropsicológico de la dismorfia muscular y del trastorno obsesivo-compulsivo. Universitas Psychologica, 17(3), 1-10. https://doi.org/10.11144/Jav eriana.upsy17-3.endm

\section{RESUMEN}

El objetivo de este estudio fue explorar y comparar las funciones ejecutivas (FE) y la memoria no verbal de un grupo de varones fisicoconstructivistas con sintomatología de dismorfia muscular (DM), con un grupo de varones que presentan sintomatología del trastorno obsesivo-compulsivo (TOC) y un grupo de control. Aplicando pruebas neuropsicológicas se encontró que los fisicoconstructivistas muestran una capacidad limitada de las FE en el control inhibitorio y flexibilidad cognitiva, con déficits visuoespaciales moderados en la memoria no verbal, mientras que el grupo con TOC presentó mayores deficiencias de rendimiento en las mismas habilidades. Se sugiere realizar más estudios para evitar un conocimiento fragmentado o contradictorio entre la DM y el TOC.

Palabras clave

funciones ejecutivas; memoria no verbal; fisicoconstructivistas; dismorfia muscular; trastorno obsesivo-compulsivo.

\begin{abstract}
The objective of this study was to explore and compare Executive Functions (EF) and non-verbal memory of a group of male bodybuilders who have symptoms of Muscle Dysmorphia (MD) with a group of men who have Obsessive Compulsive Disorder symptoms (OCD) and a control group. It was found by administering neuropsychological tests that the bodybuilders show a limited capacity of EF in the inhibitory control and cognitive flexibility, also moderate visuospatial deficits in nonverbal memory, whereas the group with OCD had higher performance deficiencies in the same skills. It is suggested to conduct more studies to avoid fragmented or contradictory knowledge between MD and OCD.

Keywords
\end{abstract}


executive functions; nonverbal memory; bodybuilders; muscle dysmorphia; obsessive-compulsive disorder.

En las últimas décadas se ha incrementado cada vez más el número de varones preocupados o insatisfechos con su imagen corporal (Grieve, 2007). Esta condición con frecuencia los motiva a realizar ejercicios de fuerza, concretamente halterofilia, levantamiento de pesas y/o fisicoconstructivismo (González, 2008), con la finalidad de ganar masa muscular (Grieve, 2007). Un subgrupo de estos varones insatisfechos puede desarrollar una preocupación patológica conocida como dismorfia muscular (DM), cuya edad de inicio se ubicaría alrededor de los $19.4 \pm 3.6$ años (Olivardia, 2001).

La DM es un trastorno que se caracteriza por una preocupación excesiva sobre la idea de no tener un cuerpo suficientemente musculoso, lo que conduce a un deterioro en las actividades sociales y laborales por la necesidad de ejercitarse (American Psychiatric Association [APA], 2013). Actualmente, la DM se incluye como un trastorno relacionado con el trastorno obsesivo-compulsivo (TOC), específicamente, dentro de la clasificación diagnóstica del trastorno dismórfico corporal (TDC), debido a que comparten sintomatología clínica como la excesiva preocupación por la imagen corporal, comportamientos o actos mentales repetitivos y deterioro social (APA, 2013). De forma complementaria, la DM es considerada como parte del espectro obsesivo-compulsivo, ya que su asociación sintomatológica se centra en un comportamiento crónico motivado por una obsesión (Compte \& Sepúlveda, 2014) y por la presencia de pensamientos intrusivos y de conductas de compensación (Rodríguez-Molina, 2007).

Sin embargo, surge la controversia sobre su clasificación al comparar personas que presentan DM con pacientes con TDC, ya que en la DM se presenta mayor probabilidad de practicar ejercicio físico de forma compulsiva (levantamiento de pesas), restricciones alimentarias, peor calidad de vida, mayor probabilidad de cometer intentos suicidas, de consumir esteroides y mayor prevalencia en trastornos por consumo de sustancias (Pope et al., 2005). Otra postura afirma que la DM se relaciona más estrechamente con los trastornos del comportamiento alimentario (TCA), por presentar un perfil patológico similar en cuanto al perfeccionismo, presencia de obsesiones, rasgos anhedónicos, preocupaciones sostenidas y relacionadas significativamente con la imagen corporal, la dieta y el ejercicio físico (Murray et al., 2012). Una tercera perspectiva menciona que la DM podría relacionarse con el TOC, debido a la presencia de obsesiones, compulsiones y la ansiedad asociada, que se limitan a la realización de entrenamientos rigurosos y dietas hipercalóricas rígidas, esteroides anabólicos y otras drogas (Olivardia, 2001). De manera similar, Maida y Armstron (2005) afirman que los síntomas de la DM están más asociados con los síntomas del espectro TOC que con los de un trastorno somatomorfo.

En esta misma línea de ideas, la relación entre la DM y el espectro TOC parece consistente debido a las evidencias mostradas en varios estudios. Pope et al. (2000) en uno de sus trabajos concluyen lo siguiente: 1) sujetos con DM presentan importantes síntomas obsesivos, por ejemplo, el 50 \% señaló la dedicación de más de tres horas diarias pensando en sus músculos y 2) en una muestra de culturistas con DM, el $54 \%$ mostró poco o nada de control sobre su necesidad y conducta compulsiva de hacer levantamiento de pesas o mantener regímenes alimentarios y el 29 \% presentó desórdenes de ansiedad. De igual forma, Kouri, Pope, Katz y Oliva (1995) avalan que componentes del TOC se han visto en porcentajes que varían desde el $28 \%$ al 60 $\%$ en varones y del $3 \%$ en mujeres con DM que practican fisicoculturismo. En otro estudio, también se encontró que los fisicoconstructivistas se caracterizan por tener una personalidad rígida, con matices obsesivo-compulsivos muy marcados en comparación con otros usuarios de gimnasio (Arbinaga \& Caracuel, 2006). No obstante lo señalado, se precisa que la DM no es un TOC, si bien existe un componente obsesivo; a diferencia del TOC, el varón con DM tiene un pensamiento y una conducta menos rígidos 
(Rodríguez-Molina, 2007), aceptando siempre cambios que le conduzcan a su objetivo (nuevas dietas, ejercicio, etc.).

Poco se sabe sobre los déficits neuropsicológicos de la relación entre los fisicoconstructivistas que presentan DM con los pacientes que presentan el TOC; lo que se conoce muy bien es que en estos últimos existen algunas limitantes (Moritz, Fricke, Wagner, \& Hand, 2001), especialmente en las funciones ejecutivas y en las aptitudes visuoespaciales de la memoria no verbal (Savage, 2001). Las funciones ejecutivas (FE) son responsables de la capacidad para captar los aspectos globales de situaciones o problemas, y de utilizar esta información para priorizar objetivos, planificar y llevar a cabo la conducta de una manera estratégica en la resolución de problemas y en la adaptación al medio (Perpiñá, García, Salhi, \& Llucià, 2002). La memoria no verbal, consiste en la capacidad para almacenar y manipular imágenes visuales mediante la planeación y la organización ejecutiva de la información almacenada (Sherman et al., 2006).

Se ha encontrado que las personas con TOC utilizan un pensamiento sobreinclusivo, de manera que clasifican la realidad en exceso, centran su atención en pequeños detalles y tienen dificultad para ejecutar tareas que requieran razonamiento intuitivo y una visión global. Además, presentan un déficit en la capacidad de cambio, que se considera un reflejo de la carencia de flexibilidad cognitiva o atencional y puede relacionarse con el componente dubitativo de los síntomas.

Estos datos se derivan de la interpretación del Test de Clasificación de Cartas de Wisconsin (WCST), en combinación con la SPECT (tomografía simple por emisión de fotón simple), al encontrar un incremento en la cantidad de errores perseverativos y respuestas incorrectas, así como un mayor número de ensayos para completar cada categoría y un menor número de categorías completas. Con el SPECT, se halló una relación entre las respuestas erróneas con el flujo cerebral de la corteza frontal inferior derecha, con la corteza orbitofrontal y con el núcleo caudado (Martínez-González \& PiquerasRodríguez, 2008).
Datos similares se han encontrado en el Test de Colores y Palabras del Stroop en donde los pacientes con TOC muestran incapacidad para evadir distractores y fallos en la inhibición de respuestas aprendidas o repetitivas, cuya base anatómica es la corteza orbitofrontal (Crawford, Parker, \& McKinlay, 1992).

También hay evidencia en los sujetos con TOC sobre la presencia de un déficit en el uso de estrategias para la organización de la información visuoespacial, donde está comprometida la corteza parietal izquierda y parieto-occipital, de acuerdo al Test de la Figura Compleja de Rey-Osterrieth. Estos problemas fueron altamente predictores de la cantidad de información que se recuperaba durante el recuerdo inmediato (Savage et al., 1999). Savage (2001) supone que las deficiencias en la memoria serían secundarias a una afectación frontal, que impide un procesamiento global de la situación y por lo tanto una afectación en la codificación de la información y el posterior recuerdo.

Con base en lo anteriormente referido, fue de interés para este trabajo de investigación identificar la presencia de un deterioro neuropsicológico en la DM similar a lo encontrado en el TOC, teniendo como objetivo explorar y comparar las FE y la memoria no verbal de un grupo de varones fisicoconstructivistas con sintomatología de DM con un grupo de varones con sintomatología del TOC y un grupo control. Se planteó la siguiente hipótesis: el desempeño del grupo con sintomatología de DM presentará un rendimiento ligeramente menor que el grupo con sintomatología del TOC en las FE y en la memoria no verbal.

\section{Método}

Se trató de un estudio descriptivo, no experimental de tipo transversal.

\section{Participantes}

La muestra fue no probabilística de tipo intencional, compuesta por 90 varones con un rango de edad de 20 a 40 años, divididos 
en tres grupos: 30 fisicoconstuctivistas con sintomatología de DM con un promedio de $\bar{X}=25.2$ años de edad $(D E=4.95), 30$ sujetos con sintomatología del TOC con un promedio de $\bar{X}=25.17$ años de edad $(D E=5.71)$ y 30 estudiantes de nivel universitario con un promedio de $\bar{X}=24.96$ años de edad $(D E=$ 3.28). Todos ellos provenientes de la zona norte del D.F. y zona conurbada.

El grupo de fisicoconstuctivistas incluyó varones que entrenaban con pesas tres $\mathrm{O}$ más veces por semana, en rutinas con duración mínima de una hora y media desde aproximadamente 12 meses y sin haber participado en competencias; además que alcanzaran o rebasaran el punto de corte $(\geq$ 52; Maida \& Armstrong, 2005) de la Escala de Motivación por la Musculatura ([DMS] por sus siglas en inglés; McCreary \& Sasse, 2000). El otro grupo se conformó con aquellos sujetos que presentaron sintomatología del TOC en la Escala Yale-Brown para el Trastorno ObsesivoCompulsivo en español ([Y-BOC]; Nicolini et al., 1996). El grupo control incluyó estudiantes que no realizaran algún tipo de actividad física en gimnasios y sin sintomatología de DM ni del TOC.

En la DMS, el grupo control (GC) obtuvo una media de $\bar{X}=26.73(D E=7.3)$, lo cual indica que no presentaron obsesión por incrementar su musculatura y tampoco por practicar algún entrenamiento con pesas, además, no utilizaban suplementos alimenticios. El grupo con sintomatología del TOC (GTOC) tuvo una media de $\bar{X}=25.83(D E=6.44)$, resultado que lo ubicó en la misma situación del GC; mientras que el grupo de fisicoconstructivistas (GDM) presentó una media de $\bar{X}=59.16$ $(D E=4.99)$, es decir, este grupo sí presentó obsesión por incrementar su musculatura y por el entrenamiento, conjuntamente, usaban suplementos alimenticios. En la Y-BOC el GC obtuvo una media de $\bar{X}=4.57(\mathrm{DE}=3.46)$, que representa una sintomatología subclínica, el grupo con GTOC presentó una media de $\bar{X}=$ $23.93(D E=14.25)$, que significa la presencia de sintomatología obsesiva y compulsiva de moderada a grave.

\section{Instrumentos}

Escala de Motivación por la Musculatura ([DMS]; McCreary $\mathcal{E}$ Sasse, 2000)

Esta escala mide actitudes y comportamientos que reflejan el grado de preocupación de la gente por incrementar su musculatura. Consta de 15 ítems tipo Likert con 6 opciones de respuesta (nunca $=1$ a siempre $=6)$. El punto de corte es $\geq 52$ (Maida \& Armstrong, 2005), de modo que puntuaciones altas indican mayor preocupación por la musculatura. Presenta una consistencia interna de $\alpha=0.83$ para varones mexicanos; de la escala se derivan tres factores (Escoto et al., 2007): Obsesión por incrementar la musculatura $(\alpha=0.85)$, Uso de suplementos alimenticios $(\alpha$ $=0.68)$ y Obsesión por el entrenamiento $(\alpha=$ $0.53)$.

Escala de Yale Brown para el Trastorno Obsesivo Compulsivo en español (Y-BOC)

Presenta una confiabilidad en un margen de 0.84 a 0.87 en pacientes mexicanos (Nicolini et al., 1996). Este instrumento cuantifica únicamente la sintomatología específica del trastorno obsesivo-ompulsivo sin enfocarse en su contenido. Consta de 12 reactivos tipo Likert; los seis primeros califican la intensidad de las obsesiones, mientras que los seis restantes califican la intensidad de las compulsiones. Cada reactivo se califica con 0 (sin sintomas) a 4 puntos (sintomatología extrema), por lo tanto, el intervalo de margen es de 0 a 40 puntos según la severidad de los síntomas: $0-7=$ subclínico, $8-15=$ leve, $16-23=$ moderado, $24-31=$ grave y $32-40$ $=$ muy grave. 
Test de Colores y Palabras de Stroop (Golden, 1994)

Evalúa la habilidad de atención selectiva, la inhibición cognitiva y la resistencia a la interferencia. Consta de tres láminas y se obtienen cuatro puntuaciones principales: 1) Número de palabras leídas correctamente en la primera lámina (P: evalúa la velocidad en la lectura de palabras); 2) Número de denominaciones correctas al color de equis impresas en la segunda lamina (C: evalúa la velocidad en la identificación de colores); 3) Número de denominaciones correctas al color de nombres de colores impresos en la tercera lamina (PC: evalúa la resistencia a la interferencia), siendo esta parte incongruente entre el significado y el color de la palabra y 4) Interferencia (proceso de una respuesta automática que domina sobre una respuesta controlada). La prueba muestra una fiabilidad test-retest de 0.9 para la primera lámina, 0.83 para la segunda y 0.91 para la tercera lámina (Spreen \& Strauss, 1991).

Test de Clasificación de Tarjetas de Wisconsin ([WCST]; Heaton, Chelune, Talley, Kay, 8 Curtiss, 2001)

La prueba mide el razonamiento abstracto y la función ejecutiva que requiere la capacidad de cambio de reglas, habilidad para desarrollar y mantener las estrategias de solución de problemas que resultan adecuadas para conseguir un objetivo a través de condiciones que implican cambios de estímulos. El WCST requiere estrategias de planificación, indagaciones organizadas y utilización del feedback ambiental para cambiar esquemas, orientación de la conducta hacia el logro de un objetivo y modulación de las respuestas impulsivas. Consiste en emparejar tarjetas con criterios de clasificación (color, forma y número). La prueba tiene un coeficiente de confiabilidad de 0.71 para el número de errores, 0.53 para respuestas perseverativas, 0.52 para errores perseverativos y 0.72 para errores no perseverativos.

Test de Copia y de Reproducción de Memoria de Figuras Geométricas Complejas (Rey, 1999)

La prueba mide la capacidad de organización, la planificación de estrategias para la resolución de problemas, la memoria visual y la capacidad visuoconstructiva. En el presente estudio, el test se utilizó para evaluar la memoria no verbal. La prueba consiste en copiar una figura con el modelo a la vista; transcurridos 15 minutos, se reproduce sin tenerla a la vista. Se califican 18 unidades hasta con dos puntos cada una, siempre y cuando el dibujo sea correcto en forma y ubicación, también se mide el tiempo de reproducción tanto en la copia como en la parte de memoria. El instrumento tiene una confiabilidad para México de 0.83 en la copia y 0.78 en la memoria (Cortés, Galindo, \& Salvador, 1996).

\section{Procedimiento}

Una vez que se obtuvo el consentimiento informado por parte de los participantes, se procedió con la aplicación de los instrumentos de forma individual en el siguiente orden: DMS, Y-BOC, Stroop, WCST y Figura Compleja de Rey. Su aplicación y calificación fue de acuerdo a los parámetros de cada una de las pruebas y se utilizaron las puntuaciones directas para su respectivo análisis. Las evaluaciones fueron realizadas por cuatro experimentadoras entrenadas para ello, durante una sesión en un espacio protegido de posibles interrupciones.

\section{Análisis de datos}

Para el análisis estadístico de los resultados, se utilizó el SPSS versión 15. Se realizó el análisis descriptivo (media y desviación estándar) para determinar la sintomatología (DM y TOC) y el nivel de funcionamiento cognitivo ( $\mathrm{FE} y$ memoria no verbal) de GDM, GTOC y GC. 
También se llevó a cabo un análisis de varianza de un factor para determinar las diferencias de las FE y en la memoria no verbal entre los grupos. Además se determinó el tamaño del efecto con la $d$ de Cohen para comparar el GDM con el GTOC.

\section{Resultados}

En el Test de Stroop se encontraron diferencias estadísticamente significativas en las tareas de lectura de palabras y de denominación de colores, en las que el GC y el GTOC obtuvieron puntuaciones similares; el GDM obtuvo un peor desempeño que los otros dos grupos. En la tarea de palabra-color y en la puntuación de interferencia, los tres grupos presentaron diferencias no significativas (Tabla 1).

Tabla 1

Medias de aciertos e interferencia por grupo en el Test de Stroop

\begin{tabular}{|c|c|c|c|c|c|c|c|c|}
\hline \multirow{3}{*}{ Tareas } & \multicolumn{6}{|c|}{ Grupo } & \multirow{3}{*}{ F } & \multirow{3}{*}{ Eta } \\
\hline & \multicolumn{2}{|c|}{ GC } & \multicolumn{2}{|c|}{ GTOC } & \multicolumn{2}{|c|}{ GDM } & & \\
\hline & Media & $D E$ & Media & $D E$ & Media & $D E$ & & \\
\hline Palabra & 92.1 & 22.26 & 92.3 & 21.88 & 49.06 & 17.34 & 43.76 ** & 0.5 \\
\hline Color & 64.5 & 13.53 & 68.86 & 12.49 & 38.5 & 13.25 & $47.1 * *$ & 0.52 \\
\hline Palabra-color & 42.1 & 14.37 & 40.36 & 7.66 & 40.2 & 12.26 & 0.24 & 0 \\
\hline Interferencia & 0.64 & 12.05 & 0.1 & 7.61 & 1.3 & 12.05 & 0.09 & 0 \\
\hline
\end{tabular}

En el WCST se encontraron diferencias estadísticamente significativas en todas las tareas. La tendencia de los resultados apunta a que el rendimiento del GC fue mejor que la de los otros dos grupos en la mayoría de la tareas: respuestas correctas, errores, errores no perseverativos, nivel conceptual y categorías completas, solo en errores perseverativos fue similar al GDM. El caso del GTOC fue el que tuvo peor desempeño que el GDM, por presentar menos respuestas correctas y categorías completas, más errores, errores perseverativos y respuestas perseverativas, sin embargo, el GDM cometió más errores no perseverativos (Tabla 2).
Tabla 2

Medias por grupo en el Test de Wisconsin

\begin{tabular}{|c|c|c|c|c|c|c|c|c|}
\hline \multirow{3}{*}{ Tareas } & \multicolumn{6}{|c|}{ Grupo } & \multirow{3}{*}{$F$} & \multirow{3}{*}{ Eta } \\
\hline & \multicolumn{2}{|c|}{$\mathrm{GC}$} & \multicolumn{2}{|c|}{ GTOC } & \multicolumn{2}{|c|}{ GDM } & & \\
\hline & Media & $D E$ & Media & $D E$ & Media & $D E$ & & \\
\hline Respuestas correctas & 93.57 & 14.65 & 67.34 & 15.96 & 72.67 & 15.03 & $24.64^{m * *}$ & 0.36 \\
\hline Errores & 34.47 & 14.71 & 60.66 & 16.09 & 55.2 & 15 & $24.3^{* * *}$ & 0.36 \\
\hline Respuestas perseverativas & 30.1 & 12.7 & 47.69 & 20.35 & 22.27 & 10.85 & $21.76^{\text {w* }}$ & 0.33 \\
\hline Errores perseverativos & 19.17 & 15.52 & 30.86 & 13.98 & $19.1^{\mathrm{a}}$ & 10.99 & $7.24^{* * *}$ & 0.14 \\
\hline Errores no perseverativos & 17.4 & 13.97 & 28.52 & 12.24 & 35.73 & 16.38 & $12.47 * *$ & 0.22 \\
\hline Nivel conceptual & 82.73 & 19.79 & 49.24 & 18.85 & 55.7 & 19.19 & 25.21 ** & 0.37 \\
\hline Categorias completas & 6.73 & 2.38 & 3.48 & 1.62 & 4.5 & 1.94 & $20.33 * *$ & 0.32 \\
\hline
\end{tabular}

En el Test de la Figura Compleja de Rey, se encontraron diferencias estadísticamente significativas en la tarea de copia, en el tiempo de ejecución de la copia y en el de la memoria. En ellas, el GTOC fue menos preciso y el más lento en terminar las tareas que el GC y el GDM; en la copia, estos se desempeñaron de forma similar, pero el GDM tardó más que el GC en terminar la copia; lo contario sucedió en el tiempo de ejecución en la memoria ya que el GDM fue más rápido que el GC. En cuanto a la precisión en la tarea de memoria, no se encontraron diferencias significativas en el desempeño de los tres grupos (Tabla 3).

\section{Tabla 3}

Medias por grupo en el Test de la Figura Compleja de Rey

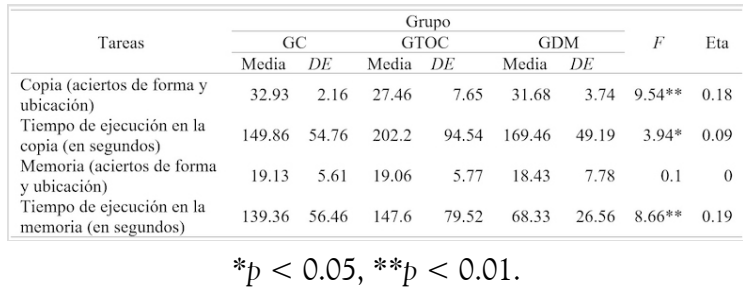

Para determinar qué tan grandes o pequeñas fueron las diferencias entre el GTOC y el GDM, se calculó el tamaño del efecto en aquellas tareas en las que se encontraron diferencias estadísticamente significativas. Los resultados mostraron discrepancias pequeñas en las dimensiones de errores, errores no perseverativos y nivel conceptual del WCST, así como en el tiempo de copia de la Figura Compleja de Rey. Discrepancias moderadas se encontraron en categorías completas del WCST y en la copia de la Figura Compleja de Rey. Las discrepancias grandes se observaron en la dimensión errores perseverativos del WCST. Las discrepancias mayores se observaron en lectura 
de palabras y denominación de color del Stroop, respuestas perseverativas del WCST y el tiempo en memoria de la Figura Compleja de Rey (Tabla 4).

\section{Tabla 4}

Tamaño del efecto con la d de Cohen comparando el grupo de GTOC con el GDM

\begin{tabular}{|lccc|}
\hline \multicolumn{1}{|c}{ Medida } & \multicolumn{2}{c}{ Medias por Grupo } & \multirow{2}{*}{$d$ de Cohen } \\
\cline { 2 - 3 } & GTOC & GDM & \\
\hline Test de Stroop & & & \\
$\quad$ Palabra & 92.3 & 49.06 & 2.19 \\
$\quad$ Color & 68.87 & 34.5 & 2.35 \\
Test de Wisconsin & & & \\
$\quad$ Respuestas correctas & 67.34 & 72.67 & 0.5 \\
Errores & 60.66 & 52.2 & 0.35 \\
Respuestas perseverativas & 47.69 & 22.27 & 1.55 \\
Errores perseverativos & 30.86 & 19.1 & 0.93 \\
Errores no perseverativos & 28.52 & 35.73 & 0.49 \\
Nivel conceptual & 49.24 & 55.7 & 0.33 \\
$\quad$ Categorias completas & 3.48 & 4.5 & 0.57 \\
Test de la Figura compleja de Rey & & & \\
Copia & 27.46 & 31.68 & 0.7 \\
Tiempo copia & 202.2 & 169.46 & 0.43 \\
Tiempo memoria & 147.6 & 68.33 & 1.33 \\
\hline
\end{tabular}

Nota: Valoración de la magnitud del tamaño del efecto (Cohen, 1988): $d=0.2$ (diferencia pequeña),$d=0.5$ (diferencia moderada) y $d=0.8$ (diferencia grande). Rosenthal (1996) añade $d=1.3$ (diferencia muy grande).

Al parecer, el GDM presentó un perfil neuropsicológico propio pero similar al del GTOC, con base en el tamaño del efecto con la $d$ de Cohen.

\section{Discusión y conclusiones}

El objetivo del presente estudio fue explorar y comparar las FE y la memoria no verbal de un grupo de varones fisicoconstructivistas con sintomatología de DM con un grupo de varones con sintomatología del TOC y un grupo control. Los resultados derivados de las pruebas neuropsicológicas aplicadas revelan que el GDM muestra una capacidad limitada de las FE que incluye el control inhibitorio y la flexibilidad cognitiva, asimismo, moderados déficits visuoespaciales en la memoria no verbal con respecto al GTOC, que presentaron mayores deficiencias.

Es posible que estas limitantes y deficiencias en el GDM disminuyan su capacidad para captar los aspectos globales de situaciones, menoscabando la utilización de esta información para organizar y planificar el almacenamiento de la información, los pensamientos y las conductas de una manera estratégica en la resolución de problemas. Se infiere que el insuficiente control inhibitorio de este grupo podría reflejar especialmente su nivel de ansiedad o angustia alto al responder más rápido que el GTOC, en la mayoría de las pruebas neuropsicológicas. Al comparar el GDM con el GC, se observó un rendimiento moderadamente inferior de los fisicoconstructivistas.

Cuando se analizaron las características clínicas y las discrepancias entre el GDM con el GTOC, se encontró, en términos generales, que ambos grupos presentaron síntomas obsesivos, compulsivos y la ansiedad asociada. Por lo tanto, se coincide con el DSM-5 al incluir a la DM como un trastorno relacionado con el TOC, sin embargo, la clasificación diagnóstica que realiza el DSM parece insuficiente por la falta de un consenso en la conformación de un perfil claro de la DM. También, se coincide con RodríguezMolina (2007) al señalar que la DM no es un TOC, ya que los pensamientos y las conductas del GDM en la pruebas neuropsicológicas fueron menos rígidos que el GTOC. Por lo tanto, se confirma la hipótesis al comprobar que el rendimiento del GDM fue ligeramente menor que el GTOC en las FE y en la memoria no verbal.

Por otro lado, los resultados de las pruebas neuropsicológicas del GTOC muestran marcadas deficiencias en varias tareas que miden las FE y la memoria no verbal; sin embargo, no parecen estar uniformemente alteradas como se ha evidenciado en la literatura, por lo que los resultados no han sido consistentes con los de otros estudios (Crawford et al., 1992; Martínez-González \& Piqueras-Rodríguez, 2008; Savage et al., 1999; Veale, Sahakian, Owen, \& Marks, 1996). La interpretación más aceptable de estas inconsistencias se relaciona con el hecho de que, probablemente, estas no sean unitarias y que dependan de circuitos frontoestriados independientes, aunque neuroanatómicamente próximos (Mataix-Cols, 2001). Estudios recientes con sujetos subclínicos han mostrado que "los déficit cognitivos del TOC podrían hacerse extensibles a individuos que 
no llegan a cumplir criterios diagnósticos para este trastorno, sugiriendo una continuidad entre los fenómenos obsesivo-compulsivos normales y patológicos y la implicación de los sistemas frontosubcorticales en la conducta obsesiva normal" (Mataix-Cols, 2001). Es posible que se conceptualice al TOC como un conjunto de enfermedades con etiología y bases neurales distintas, en lugar de una entidad nosológica unitaria (Mataix-Cols, Rauch, Manzo, Jenike, \& Baer, 1999).

Sugerencias: 1) en lugar de utilizar baterías neuropsicológicas globales rígidas, a menudo con resultados contradictorios, se sugiere emplear pocas pruebas, más sensibles a las diferencias sutiles de síntomas; 2) incluir grupos clínicos de TOC; 3) incorporar subtipos de TOC; 4) correlacionar índices neuropsicológicos con medidas de ansiedad y 5) correlacionar datos de neuroimagen con neuropsicológicos para entender mejor las bases funcionales del déficit cognitivo y conductual de ambos grupos. Por lo tanto, se requiere de estudios más finos para poder identificar un patrón de deterioro cognitivo y conductual de la DM con el fin de evitar un conocimiento fragmentado o contradictorio.

\section{Referencias}

American Psychiatric Association. (2013). Diagnostic and statistical manual of mental disorders (5th. ed.) [Manual diagnóstico y estadístico de los trastornos mentales DSM-5 (C. Garay, Trad.)]. Barcelona: Editorial Médica Panamericana.

Arbinaga, F., \& Caracuel, J. C. (2006). Perfil socio-deportivo de fisicoculturistas competidores versus no competidores. Cuadernos de Psicología del Deporte, 6(1), 7-20. Recuperado de http://revistas.um.es/c $\mathrm{pd} /$ article/view/70951/68411

Cohen, J. (1988). Statistical power analysis for the behavioral sciences (2nd ed.). Hillsdale, NJ: Lawrence Earlbaum Associates

Compte, E., \& Sepúlveda, A. (2014). Dismorfia muscular: perspectiva histórica y actualización en su diagnóstico, evaluación y tratamiento. Psicología Conductual, 22(2), 307-326. Recuperado de http://www.funda cionforo.com/pdfs/dismorfia-muscular.pdf

Cortés, S. J. F., Galindo, V. M. G., \& Salvador, C. J. (1996). La figura compleja de Rey: propiedades psicométricas. Salud Mental, 19(3), 42-48. Recuperado de http://revistasaludmental.mx/index.php /salud mental/article/view/598/598

Crawford, J. R., Parker, D. M., \& McKinlay, W. W. (1992). A handbook of neuropsychological assessment. Hove, UK: Erlbaum.

Escoto, M. C., Álvarez, G. L., Bedolla, G., Velázquez, B., Yáñez, K., \& Mancilla, J. M. (octubre, 2007). Consistencia interna y estructura factorial de la Drive for Muscularity Scale. Trabajo presentado en el XV Congreso Mexicano de Psicología, Hermosillo, Sonora.

Golden, C. J. (1994). Stroop. Test de colores y palabras. Madrid: TEA.

González, I. (octubre, 2008). Vigorexia: instrumentos para su detección. Actas del $V$ Congreso de la Asociación Española de Ciencias del Deporte. Universidad de León, España. Recuperado de http://www.cienciadeporte.com/images/ congresos/leon/salud,\%20ocio\%20y \%20recreacion/gonzalezvigorexia.pdf

Grieve, F. G. (2007). A conceptual model of factors contributing to the development of muscle dysmorphia. Eating Disorders, 15(1), 63-80. https://doi.org/10.1080/1064026060 1044535

Heaton, R. K., Chelune, G. J., Talley, J. L., Kay, G. G., \& Curtiss, G. (2001). Test de Clasificación de Tarjetas de Wisconsin (2. ed.). Madrid: TEA.

Kouri, E. M., Pope, H. G, Katz, D. L., \& Oliva, P. (1995). Fat-free mass index in users and nonusers of anabolic-androgenic steroids. Clinical Journal of Sports Medicine, 5(4), 223-228.

Nicolini, H., Herrera, K., Paéz, F., Sánchez de Carmona, M., Orozco, B., Codeiro, G., \& de la Fuente, J. R. (1996). Traducción al español y 
confiabilidad de la escala Yale-Brown para el trastorno obsesivo-compulsivo. Salud Mental, 19(Supl. 3), 13-16. Recuperado de http://revistasaludmental.mx/index.php /salud_mental/article/view/629

Maida, D. M., \& Armstrong, S. L. (2005). The classification of muscle dysmorphia. International Journal of Men's Health, 4(1), 73-91. Recuperado de https://pdfs.semanticscholar.org/ecea/bf 57257f4543c8dacedf5d8c387b9087f25e.pd f

Martínez-González, A. E., \& PiquerasRodríguez, J. A. (2008). Actualización neuropsicológica del trastorno obsesivocompulsivo. Revista de Neurología, 48(10), 625- 625. Recuperado de https://www.neur ologia.com/articulo/2008064

Mataix-Cols, D. (2001). Neuroimagen y neuropsicología del trastorno obsesivocompulsivo: avances recientes. Psiquiatría Biológica, 8(2), 54-63. Recuperado de http://www.elsevier.es/es-revista-psiquia tria-biologica-46-articulo-neuroimagen-ne uropsicologia-del-trastorno-obsesivo-comp ulsivo-13013326

Mataix-Cols, D., Rauch, S. L., Manzo, P. A., Jenike, M. A., \& Baer, L. (1999). Use of factor-analyzed symptom dimensions to predict outcome with serotonin reuptake inhibitors and placebo in the treatment of obsessive-compulsive disorder. American Journal of Psychiatry, 156(9), 1409-1416. ht tps://doi.org/10.1176/ajp.156.9.1409

McCreary, D. R., \& Sasse, D. K. (2000). An exploration of the drive for muscularity in adolescent boys and girls. Journal of American College Health, 48(6), 297-304. ht tps://doi.org/10.1080/07448480009596271

Moritz, S., Fricke, S., Wagner, M., \& Hand, I. (2001). Further evidence for delayed alternation deficits in obsessive-compulsive disorder. Journal Nervous and Mental Disease, 189(8), 562-564. https://doi.org/10 $.1097 / 00005053-200108000-00012$

Murray, S., Rieger, E., Hildebrandt, T., Karlov, L., Russell, J., Boon, E. ... Touyz, S. W. (2012). A comparison of eating, exercise, shape, and weight related symptomatology in males with muscle dysmorphia and anorexia nervosa. Body Image, 9(2), 193-200. https://doi.org/10.1016/j.bodyim. 2012.01.008

Olivardia, R. (2001). Mirror, mirror on the wall, who's the largest of them all? The features and phenomenology of muscle dysmorphia. Harvard Review of Psychiatry, 9(5), 254-259. https://doi.org/10.1080/hrp.9.5.254.259

Perpiñá, S., García, L., Salhi, G., \& Llucià, T. (2002). Aspectos neuropsicológicos del trastorno obsesivo compulsivo. Revista de Neurología, 35(10), 959-963. Recuperado de https://www.neurologia.com/articulo/20 02117

Pope, H. G., Gruber, A. J., Mangweth, B., Benjamin, B., De Col, C., Jouvent, R., \& Hudson, J. I. (2000). Body image perception among men in three countries. American Journal of Psychiatry, 157(8), 1297-1301. ht tps://doi.org/10.1176/appi.ajp.157.8.1297

Pope, C. G., Pope, H. G., Menard, W., Fay, C., Olivardia, R., \& Phillips, K. (2005). Clinical features of muscle dysmorphia among males with body dysmorphic disorder. Body Image, 2(4), 395-400. https:/ /doi.org/10.1016/j.bodyim.2005.09.001

Rey, A. (1999). Test de Copia y de Reproducción de Memoria de Figuras Geométricas Complejas

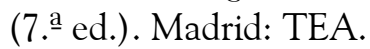

Rodríguez-Molina, J. M. (2007). Vigorexia: adicción, obsesión o dismorfia: un intento de aproximación. Salud y Drogas, 7(2), 289-308. Recuperado de http://www.redaly c.org/pdf/839/83970205.pdf

Rosenthal, J. A. (1996). Qualitative descriptors of strength of association and effect size. Journal of Social Service Research, 21(4), 37-59. https://doi.org/10.1300/J079v21n04 02

Savage, C. R. (2001). Neuropsicología del TOC: hallazgos científicos e implicaciones terapéuticas. En M. A. Jenike, L. Baer \& W. E. Minichello (Eds.), Trastorno obsesivocompulsivo: manejo práctico (pp. 254-275). Madrid: Editorial Hartcourt Brace. 
Savage, C. R., Kuether, N. J., Jenike, M. A., Brown, H. D., Baer, L., Kendrick, A. D., ... Albert, M. S. (1999). Recall and recognition memory in obsessive-compulsive disorder. Journal of Neuropsychiatry Clinical Neuroscience, 8(1), 99-103. https://doi.org/10.1176/jnp.8.1.99

Sherman, B. J., Savage, C. R., Eddy, K. T., Blais, M. A., Deckersbach, T., Jackson, S. C. ... Herzog, D. B. (2006). Strategic memory in adults with anorexia nervosa: Are there similarities to obsessive compulsive spectrum disorders? International Journal of Eating Disorders, 39(6), 468-476. https://do i.org/10.1002/eat.20300

Spreen, O., \& Strauss, E. (1991). A compendium of neuropsychological test: Administration, norms, and commentary. Nueva York. Oxford University Press.

Veale, D. M., Sahakian, B. J., Owen, A. M., \& Marks, I. M. (1996). Specific cognitive deficits in tests sensitive to frontal lobe dysfunction in obsessivecompulsive disorder. Psychological Medicine, 26(6), 1261-1269. https://doi.org/10.1017/ S0033291700035984

\section{Notas}

* Artículo de investigación. 